Review

\title{
MiR-206, a Key Modulator of Skeletal Muscle Development and Disease
}

\author{
Guoda Ma1,2, Yajun Wang ${ }^{3}$, You $\mathrm{Li}^{1}$, Lili Cui ${ }^{1}$, Yujuan Zhao ${ }^{4}$, Bin Zhao ${ }^{2}$, Keshen Li ${ }^{1}$ \\ 1. Guangdong Key Laboratory of Age-Related Cardiac and Cerebral Diseases, Affiliated Hospital of Guangdong Medical College, Zhan- \\ jiang 524001, China; \\ 2. Institute of Neurology, Guangdong Medical College, Zhanjiang 524001, China; \\ 3. Affiliated Hospital of Guangdong Medical College, Zhanjiang 524001, China; \\ 4. Department of Cardiology, the First Affiliated Hospital of Harbin Medical University, Harbin 150001, China.
}

\begin{abstract}
$\triangle$ Corresponding author: E-mail: binzhaoe@163.com (B. Zhao) or likeshen1971@126.com (K. Li). Tel \& Fax: +86 7592386949
(c) 2015 Ivyspring International Publisher. Reproduction is permitted for personal, noncommercial use, provided that the article is in whole, unmodified, and properly cited. See http://ivyspring.com/terms for terms and conditions.
\end{abstract}

Received: 2014.10.27; Accepted: 2015.01.01; Published: 2015.02.05

\begin{abstract}
MicroRNAs (miRNAs) have recently emerged as fundamental post-transcriptional regulators inhibit gene expression linked to various biological processes. MiR-206 is one of the most studied and best characterized miRNA to date, which specifically expressed in skeletal muscle. In this review, we summarized the results of studies of miR-206 with emphasis on its function in skeletal muscle development. Importantly, dysregulation of miR-206 has been linked to many disorders in skeletal muscle such as Duchenne muscular dystrophy (DMD) and amyotrophic lateral sclerosis (ALS), and circulating miR-206 has highlighted its potential as a diagnose biomarker. In addition, a mutation in the 3' untranslated region (3'-UTR) of the myostatin gene in the Texel sheep creating a target site for the miR-206 and miR-1 leads to inhibition of myostatin expression, which likely to cause the muscular hypertrophy phenotype of this breed of sheep. Therefore, miR-206 may become novel target for ameliorating skeletal muscle-related disorders and optimization of muscle quantity of domestic animals.
\end{abstract}

Key words: miR-206, skeletal muscle, development, disease

\section{Introduction}

Most skeletal muscles are derived from paraxial mesoderm cells, which further develop into somites. Skeletal muscle precursor cells that reside in somites undergo cell-fate decisions, followed by proliferation and differentiation. The precursor cells migrate along an established route to specific sites of new muscle tissue formation and differentiate into skeletal muscles [1]. This process involves complex gene expression regulatory networks, which function primarily through precise control of intercellular signaling and regulation of the expression of specific genes. Studies have shown that the myogenic regulatory factor (MRF) family and the myocyte enhancer factor-2 (MEF2) family play central roles during skeletal muscle formation and development [2-5].
In the past, our understanding of the regulation of muscle gene expression has mainly been restricted to the transcriptional level. MicroRNAs (miRNAs) are a class of non-coding RNAs involved in post-transcriptional gene silencing; they inhibit translation or promote mRNA degradation generally by associating with the 3 '-UTR of their regulatory target mRNAs. In various biological processes, miRNAs fine-tune the protein levels of key regulatory factors or act as a switch to control gene expression [6]. Post-transcriptional regulation mediated by miRNAs adds another layer of complexity to gene expression regulation and enhances its sensitivity and accuracy [7]. Recently, their role in skeletal muscle development has become increasingly clear $[8,9]$. 
The expression of numerous miRNAs is tissue-specific. The miRNAs specifically expressed in muscle tissues are termed the myomiR family and include miR-1, miR-133, miR-206, miR-208a/b, miR-499 and miR-486 [10-13]. Except for miR-206 and miR-208a, most myomiRs are expressed in both cardiac and skeletal muscles. MiR-206 is expressed specifically in skeletal muscles [10], while miR-208a is reported to be expressed predominantly in cardiac muscles [13]. The present review article summarizes the latest research progress on miR-206.

\section{The biological characteristics of miR-206}

\subsection{The chromosomal localization, structure and expression patterns of miR-206}

MiR-206 is a member of the miR-1 family, and the gene for miR-206 is located between the interleukin-17 gene and the polycystic kidney and hepatic disease 1 gene in human (chr 6), mouse (chr 1) and rat (chr 9), and their sequences are highly conserved [14, 15]. MiR-1-1 and miR-1-2 share an identical nucleotide sequence, while the sequence of miR-206 differs from miR-1-1 and miR-1-2 by four nucleotides outside of the seed region. MiR-133a-1, miR-133a-2 and miR-133b have virtually the same sequence except for a single nucleotide at the $3^{\prime}$ end $[7,14]$. The sequence similarity between the myomiRs indicates that they have the same or similar target genes. The six miR-1 family members are arranged into three homologous genes clusters, the miR-1-2/miR-133a- 1 , the miR-1-1/miR-133a-2 and the miR-206/miR-133b gene clusters [16]. Of the three clusters, the miR-206/miR-133b cluster appears late in evolution and is expressed only present in vertebrates, whereas the other two clusters are highly conserved across species from drosophila to humans. The origin of the miR-206 locus may contribute to the higher complexity of vertebrate skeletal muscle. In fact, fly and worm do not possess different fiber types, such as slow- and fast-twitch fibers, nor do they have satellite cells, as found in vertebrate skeletal muscle. Consistent with the hypothesis, it has been reported that miR-206 expression is higher in muscle composed primarily of slow-twitch/oxidative fibers i.e, soleus versus plantaris [17].

Sempere [10] et al. provided the first description of miRNAs specifically expressed in striated muscles, including miR-1, miR-133 and miR-206, and demonstrated that miR-206 displayed skeletal muscle-specific expression. During mouse embryonic development, miR-206 is expressed in skeletal myoblasts in somites, limb buds and head muscles. A very low level of miR-206 expression has been first detected in mice at 9.5 days post-coitum (dpc), and then, beginning around 11.5-12.5 dpc, miR-206 expression begin to significantly increase [18]. Post-natal expression of miR-206 reaches a peak value $3 \mathrm{~d}$ after birth, and then starts to decline [13]. Similarly, miR-206 expression is limited to somites in fish, frog and chicken embryos [19]. This expression pattern of miR-206 indicates that miR-206 plays an essential role in muscle differentiation and cell formation during embryonic development.

\subsection{Regulation of miR-206 expression}

Skeletal myoblast development is regulated by growth factors in the environment in which the myoblasts reside [1]. Studies have shown that transforming growth factor- $\beta$ (TGF- $\beta$ ) regulate muscle differentiation through inhibiting miR-206 expression [20]. Rachagani et al reported that the expression of miR-206 is significantly higher in the skeletal muscle of myostatin knockout mice compared to that of wild type mice, which indicates that myostatin may regulates the expression of miR-206 [21]. miR-206 contains two promoters, the proximal promoter driving expression of miR-206, and the distal one responsible for the transcription of the whole transcript containing miR-206, miR-133b and a muscle specific long non-coding RNA, which is named as linc-MD1 [22]. The myogenesis-related transcription factors MyoD, Myogenin (Myog) and Mef2 bind to the upstream regions of the miR-206 locus, ensure and maintain its skeletal muscle tissue-specific expression [19, 23, 24] (Figure 1).

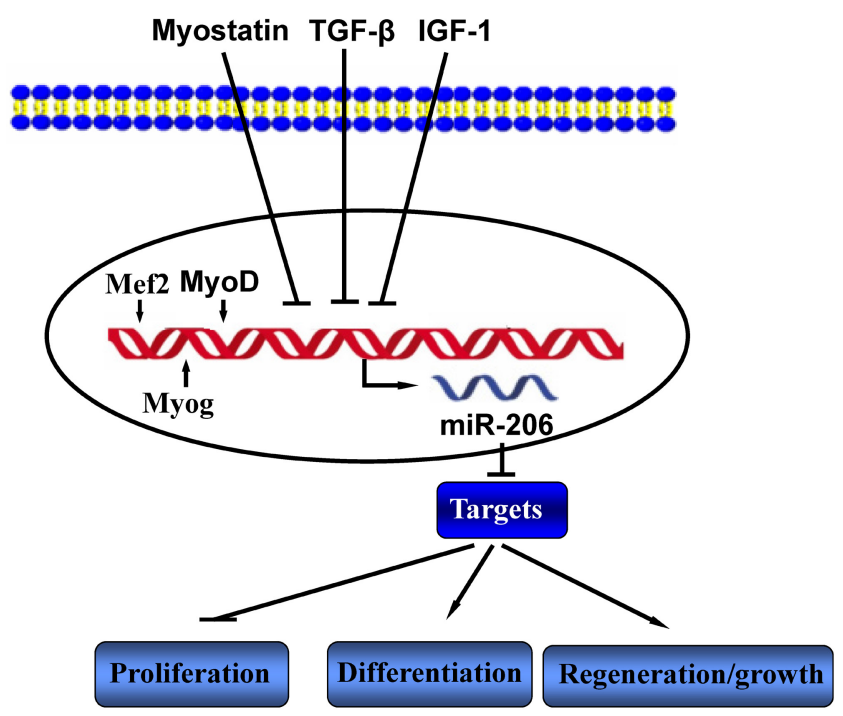

Figure 1. The miR-206 regulators and functions in skeletal muscle. $\longrightarrow$ indicate upregulation or activation; -1 indicate downregulation or inhibition. 


\section{The functions of miR-206 in skeletal muscle development and the underlying molecular mechanisms}

\section{1 miR-206 and skeletal myoblast differentia- tion}

When cultured in vitro, the mouse myoblast cell line $\mathrm{C} 2 \mathrm{C} 12$ is capable of simulating the in vivo process of skeletal muscle cell proliferation and differentiation. In serum-containing growth medium, the myoblasts maintain a proliferating status. Removal or reduction of the serum from the culture medium induces the $\mathrm{C} 2 \mathrm{C} 12$ myoblasts to form terminally differentiated myotubes. In the process of inducing C2C12 myoblast differentiation into myotubes, miR-206 expression is significantly upregulated [10]. Overexpression of miR-206 in C2C12 cells blocks cell cycle progression and induces myotube formation, whereas the inhibition of miR-206 expression produces opposite results [25]. These findings indicate that miR-206 plays an important regulatory role in skeletal muscle differentiation. The functions of miRNAs are achieved primarily through their targets. A number of downstream target genes of miR-206 have been verified, which, to a certain extent, illustrate the molecular mechanism underlying the promotion of skeletal muscle differentiation by miR-206 (Figure 2).

\subsubsection{DNA polymerase alpha 1 (Pola1)}

Cell cycle arrest and decreased DNA synthesis are the two key events in the differentiation process.

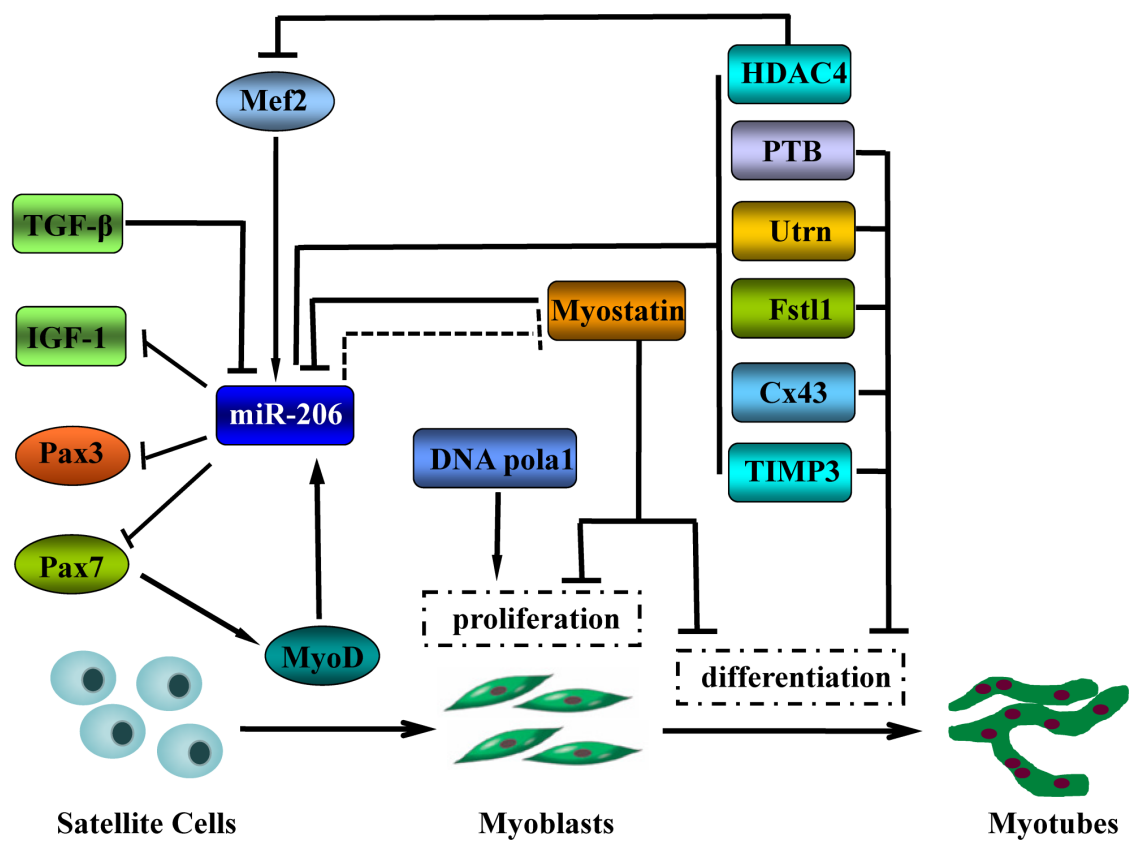

Figure 2. The functions of miR-206 in skeletal muscle cell development and the underlying molecular mechanisms. $\longrightarrow$ activation; $\longrightarrow$ inhibition; $\cdots \cdot \cdots \cdot \cdot$ inhibition in Texel sheep.
Pola1 is the largest subunit of DNA polymerase and is responsible for cellular DNA synthesis. Kim [25] et al. found that miR-206 negatively regulates the translation of DNA Pola1 and inhibits DNA synthesis in the myoblast cell line $\mathrm{C} 2 \mathrm{C} 12$ through mediating the degradation of Pola1 mRNA, thereby ultimately promoting muscle differentiation. This was the first example for which miRNA was discovered to exert a direct effect on DNA replication. Inhibition of DNA synthesis occurs prior to cell cycle arrest, and Pola1 plays an important role in cell quiescence. Therefore, the activity of miR-206 links the quiescent stage of cells to the cell differentiation process.

\subsubsection{Paired box $3(\operatorname{Pax} 3)$ and $\operatorname{Pax} 7$}

The transcription factors Pax3 and Pax7 are capable of inhibiting satellite cell apoptosis and maintaining satellite cell or myoblast survival and proliferation, and they are marker genes for quiescent satellite cells [26-28]. During skeletal muscle development, they are also able to block early differentiation of myoblasts, the onset of myogenesis will be delayed when they are not inhibited in time. They are direct regulatory targets of miR-206, and timely downregulation of their expression following miR-206 transcription is required for satellite cells or primary myoblast successfully transition from proliferation to differentiation $[29,30]$.

\subsubsection{Connexin $43(\mathrm{C} \times 43)$}

Cx43 is the major component of gap junctions, which allow the passage of signaling molecules and metabolites and also coordinate differentiation and contractility in the process of embryonic skeletal muscle fusion and differentiation [31]. Therefore, Cx43 plays an essential role in muscle regeneration and in vitro differentiation. A study conducted by Anderson [16] et al. revealed the presence of two conserved target sites for the seed sequence of miR-206 in the 3' -UTR of the gap junction protein $\mathrm{Cx} 43$. Therefore, the translation of $\mathrm{Cx} 43$ mRNA is inhibited by miR-206. It is thought that the down-regulation of $\mathrm{Cx} 43$ during perinatal muscle development is necessary for the proper formation of the mature neuromuscular junction (NMJ) [16]. In addition, miR-206 reduces the communication between the 
growing muscle fibers through downregulation of Cx43 expression, thus promoting muscle cell differentiation.

\subsection{4 utrophin (Utrn) and follistatin-like 1 (Fstll)}

A study conducted by Rosenberg [32] et al. showed that miR-206 mediate MyoD-dependent inhibition of Utrn and Fstl1 genes in myoblasts. In this case, MyoD activates the expression of miR-206, which in turn inhibits Utrn and Fstl1 gene expression post-transcriptionally. The two genes possess the function of maintaining the proliferating status of myoblasts. MiR-206 suppresses excessive muscle growth through its target genes, Fstl1 and Utrn.

\subsubsection{Histone deacetylase 4 (HDAC4)}

Histone deacetylases play a role in the process of chromatin remodeling. In particular, HDAC4 is highly expressed in cardiac and skeletal muscles. HDAC4 is a repressor of the pro-differentiation transcription factor MEF-2 and is capable of inhibiting myoblast differentiation. A study has shown that miR-206 promotes skeletal muscle cell differentiation through inhibiting HDAC4 [20].

\subsection{6 polypyrimidine tract-binding protein (PTB)}

MiR-206 inhibits the translation of PTB and its homolog $\mathrm{NPTB}$ [33]. nPTB and PTB belong to a class of regulatory factors that inhibit the alternative splicing of various transcripts. MiR-206 exerts its effects on the genetic program of muscle differentiation by regulating $\mathrm{nPTB} / \mathrm{PTB}$.

In addition, in a mouse model of skeletal muscle myogenesis, miR-206 upregulates tumor necrosis factor a (TNFa), activates the mitogen-activated protein kinase (MAPK) pathway, induces the expression of muscle-related genes and ultimately promotes myogenesis through downregulation of the metalloproteinase inhibitor 3 (TIMP3) [34], a physiological inhibitor of myogenesis. Thus, it can be concluded that miR-206 achieves the function of promoting myogenic differentiation by affecting DNA polymerase, a gap junction protein, muscle structural proteins and transcription factors and through regulating enzyme activity.

\section{2 miR-206 and muscle satellite cell differen- tiation and regeneration}

Satellite cells are stem cells present in adult skeletal muscles responsible for post-natal muscle growth and muscle regeneration from injury, which originate from myotome and dermomyotome progenitor cells in the dorsal portion of the somites [35]. Satellite cells are located between the sarcolemma and the basement membrane of muscle fibers and require Pax3 and Pax7 for their specification [36]. Further- more, these progenitor cells migrate to the developing limb buds requires Pax3 induction of c-Met gene expression. Normally, satellite cells maintain a quiescent state. Once stimulated, they enter the cell cycle and differentiation program. Following muscle injury, miR-206 expression is upregulated in satellite cells, and expression of the downstream target genes Pax3, Pax7 and c-Met is inhibited. Timely downregulation of the expression of the miR-206 target genes following miR-206 transcription is required for satellite cell differentiation [29,37]. In addition, MyoD acts as a transcriptional regulatory factor for miR-206 in the process of skeletal muscle cell differentiation. MiR-206 inhibits the expression of Pax7 and promotes the function of MyoD, thus establishing a positive regulatory feedback loop. Upregulation of miR-206 by MyoD further suppresses Pax7, thereby promoting muscle cell differentiation [37].

MiR-206 expression is elevated in regenerated muscles that are induced by cardiotoxin [33]. Nakasa et al. have investigated the effect of local injection of myomiRs containing miR-206 on muscle tissue regeneration in a rat model of skeletal muscle injury and regeneration [38], and the morphological and physiological indicators of muscle regeneration were examined. Enhanced muscle regeneration was observed in the group of rats injected with miRNAs at one week after the injury. In addition, miRNAs injection effectively inhibited muscle fibrosis. Based on the results, it is speculated that miR-206 plays a positive role in the treatment of skeletal muscle injury.

\subsection{Role of miR-206 in muscular hypertrophy and atrophy}

Skeletal muscle is the most abundant tissue and has a remarkable ability to rapidly adjust to changes in locomotion and metabolism. Adult skeletal muscle mass is mainly determined by a balance between protein synthesis and degradation. Skeletal muscle mass is gain (hypertrophy) or loss (atrophy) in response to a variety of signaling pathways. It is well known that insulin-like growth factor-1 (IGF-1) signaling plays critical positive roles in skeletal muscle growth. By contrast, myostatin pathways suppress muscle growth and/or induce muscle atrophy [39]. Several evidences demonstrated that miR-206 involved in modulating skeletal muscle mass. First, inhibition of miR-206 promotes tilapia skeletal muscle growth through upregulation of IGF-1 expression [40] and robustly increases C2C12 myotube width [41]. Second, McCarthy [17] et al. studied load-induced skeletal muscle hypertrophy in mice and found that the expression of the primary miR-206 transcript is significantly increased. Third, miR-206 is highly expressed in newly formed muscle fibers, indicating 
that miR-206 may be involved in muscle regeneration and maturation in muscle atrophy [42]. Notably, a genetic link has connected miR-206 function to muscular hypertrophy. Texel sheep display the muscular hypertrophy phenotype. Genetic linkage analysis has shown that the quantitative trait locus is located in a region of chromosome 2 that contains the myostatin gene. In mice, cattle and humans, myostatin deficiency leads to the double-muscling phenotype [43-45]. Therefore, myostatin is considered to be the candidate gene that causes muscular hypertrophy in Texel sheep. However, no polymorphisms have been detected in the coding region of the myostatin gene. Interestingly, a point mutation occurs in the 3'-UTR of the myostatin gene in which the base $G$ is mutated into $\mathrm{A}$, leading to the generation of target sites for miR-206 and miR-1. As a result, the expression level of myostatin protein is reduced, and the muscle mass of Texel sheep is increased significantly [46]. The findings reveal the genetic basis of muscular hypertrophy in Texel sheep, integrating quantitative genetic and molecular genetic mechanisms. The findings not only provide a clear example of the effect of miR-206 on skeletal muscle cell phenotype but also offer new perspectives on phenotypic changes induced by a single nucleotide polymorphism in miRNA target. The question of whether the same mechanism also exists in other animals has drawn research interest from workers in the fields of animal genetics and breeding. Further miRNA studies will provide novel molecular biological approaches to improve the meat yield and quality of livestock and poultry.

\section{3. miR-206 and muscle diseases}

MiRNA-206 is unique among the myoRNAs in that it highly is and exclusively expressed in skeletal muscle, which has a profound effect on myogensis. Recently, aberrant miR-206 expression has been observed during muscle disorders, including DMD, ALS and rhabdomyosarcoma (RMS) (Table 1).

\section{1 miR-206 and DMD}

DMD is a lethal form of muscular dystrophy that commonly occurs in adolescents. The etiology of
DMD involves muscle fiber damage and muscle atrophy induced by the loss of the structural protein dystrophin at the plasma membrane of muscle fibers [47]. Several reports have demonstrated the potential role of miR-206 in the post-transcriptional inhibition of utrophin [32, 50, 51]. However, Amirouche [15] and coworkers discovered that miR-206 overexpression in cultured $\mathrm{C} 2 \mathrm{C} 12$ cells and dystrophic muscle fibers causes upregulation of endogenous utrophin levels. Utrophin is paralogous to dystrophin [27, 46], which may replace dystrophin functionally in skeletal muscles and limit disease progression [52]. Thus, miR-206-mediated increasing utrophin expression in skeletal muscles may serve as a potential therapeutic approach for DMD.

The mdx mouse deficient in the dystrophin gene is a common model utilized to study the pathogenesis of muscular dystrophy and the mechanisms of skeletal muscle injury and regeneration. Greco [48] et al. examined differentially expressed miRNAs by comparing miRNA expression in skeletal muscle tissues between $\mathrm{mdx}$ mice and wild type mice as well as between DMD patients and normal healthy individuals. They found that the expression of miR-206 is elevated in mdx mice and in patients with DMD. Further, in situ hybridization analysis showed that miR-206 is expressed mainly in newly formed myotubes or regenerated fibers [42].

Recently, a study conducted by Liu et al. confirmed that miR-206 plays a protective role in muscular dystrophy [49]. In mdx mice, muscle injury leads to the activation of miR-206, which promotes the formation of new muscle fibers. The miR-206 knockout mdx mice show early onset of the muscular dystrophy phenotype, and their muscle regeneration and differentiation are delayed. In addition, the integrity and function of muscle fibers are impaired due to muscle fibrosis, fatty infiltration and muscle fiber mineralization. These results indicate that miR-206 plays an essential role in the pathological process of skeletal muscle injury and regeneration. Therefore, miR-206 can be used as an important therapeutic target for the treatment of DMD.

Table 1. Over view of miR-206 roles in skeletal muscle related diseases

\begin{tabular}{lll}
\hline Disease & Signification of miR-206 & Related target(s) \\
\hline DMD & Elevated levels in serum are a potential drug target and biomarker for DMD & utrophin \\
RMS & $\begin{array}{l}\text { miR-206 is not expressed in RMS cells, and miR-206 blocks RMS cells proliferation, } \\
\text { induces apoptosis and inhibits invasion }\end{array}$ & Met, MSC \\
miR-206 delays the onset and progression of ALS by promoting the regeneration of & HDAC4, BDNF, MEF2 & [42, 48-52, 59-62] \\
& neuromuscular synapses & [55-58]
\end{tabular}




\section{2 $\mathrm{miR}-206$ and RMS}

RMS is a malignant soft tissue tumor caused by a low degree of differentiation of skeletal muscle cells. Taulli [53] et al. found that miR-206 is not expressed in RMS cells. Moreover, the expression of miR-206 cannot be induced even under serum-free conditions. Re-expression of miR-206 in RMS cells blocks cell proliferation, induces apoptosis and inhibits invasion. Furthermore, overexpression of miR-206 in nude mice with RMS promotes cell differentiation and prevents tumor growth. The c-Met gene, which encodes the receptor tyrosine kinase c-Met, is a target gene of miR-206. c-Met is expressed at a low level in the initial stage of myogenic differentiation and becomes highly expressed in various tumors including RMS. Expression of the phosphorylated, constitutively active c-Met from the proto-oncogene devoid of the 3'-UTR in RMS cells expressing miR-206 abolishes miR-206mediated differentiation [48]. Therefore, c-Met is believed to be a key target molecule through which miR-206 exerts its anti-cancer effect in RMS. Downregulation of miR-206 expression and the subsequent increase in c-Met expression may be pathological events contributing to RMS, while miR-206 overexpression-induced RMS differentiation may be employed as an effective therapeutic strategy for treating patients with RMS. In addition, the transcription factors RUNX1 and ZNF238 induce RMS cell differentiation through upregulation of miR-206. Furthermore, miR-206 inhibits the activity of transcription factor Musculin (MSC) while MSC blocks the MyoD binding sites in the miR-206 promoter through binding to the E-proteins, thus generating a negative feedback loop and regulating the differentiation of RMS [54]. These results indicate that miR-206 may serve as a master regulator of gene expression to maintain muscle organization and prevent the incidence of tumors. MiR-206 re-expression is of great significance for the clinical treatment of RMS and provides a novel approach to handcuff oncogenes and arrest tumor development.

\section{3 $\mathrm{miR}-206$ and ALS}

Skeletal muscle must be properly innervated to properly perform its function by cooperation with axons and creating NMJ. ALS is the most common motor neuron disease in adults. ALS is caused initially by progressive degeneration of motor neurons controlling muscle movements, which eventually leads to muscle atrophy and paralysis. Williams et al. utilized the G93A-SOD1 transgenic mice as the mouse model of ALS in an attempt to determine the changes in miRNA expression during ALS progression [50]. The authors have found that the expression of miR-206 increases significantly in synaptic regions of muscle fibers of the mouse model of ALS. There, miR-206 as a key regulator of bidirectional signaling between skeletal muscle fibers and nerves, miR-206 is capable of sensing damage or loss of motor neurons and promoting the regeneration of functional synapses between individual neurons in the muscles to attenuate muscle injury. Overexpression of miR-206 in a mouse model of ALS delays the onset and progression of ALS by promoting the regeneration of neuromuscular synapses. Although the initial onset of ALS is the same between miR-206 knockout G93A-SOD1 mice and G93A-SOD1 mice, nerve regeneration was delayed in the miR-206 knockout mice. The disease develops faster in miR-206 knockout mice, and the survival rate of miR-206 knockout mice is lower. The benefit conferred to ALS mice by miR-206 was proposed to occur through suppression of HDAC4 mRNA, a regulatory factor that controls gene expression in nerves and muscles. In contrast, knockout of HDAC4 in mice exhibited enhanced reinnervation. HDAC4 in turn inhibits the expression of fibroblast growth factor binding protein 1 (fgfbp1), a secreted factor. fgfbp1 acts a retrograde signal to the motor nerve, promoting reinnervation, which is believed to enhance the activities of fibroblast growth factors (FGF7, 10, and 22) on distal motor neuron terminals [55]. In addition to HDAC4, it also validated that miR-206 negative regulated MEF2 [56], brain-derived neurotropic factor (BDNF) and its receptor (p75NTR) [57] to facilitate mutual communication between motoneuron axons and muscle fibers. Therefore, miR-206 has important functions at the NMJ and the molecules in the signaling pathways that regulate miR-206 expression may serve as effective targets for treating ALS.

\section{4 miR-206 as a potential biomarker for mus- cle-related disorders}

Creatine kinase may serve as an indicator for the degree of muscle damage and for use as a diagnostic marker for various muscle diseases. To date, there is no serum biomarker for RMS. The expression level of miR-206 appears to be higher in RMS cells compared to in other cell lines, and the serum level of miR-206 is significantly increased in patients with RMS in comparison with non-RMS patients and healthy individuals, which indicates that miR-206 may serve as a diagnostic marker for RMS [51]. Recently, a number of studies have demonstrated that the serum levels of miR-206 are elevated in patients with DMD and ALS and in various model animals and that the serum level of miR-206 is correlated with the severity of the disease [58-62]. Another study has demonstrated that the miR-206 level in the serum of mdx mice is less affected by movement compared to creatine kinase [62]. 
Therefore, miR-206 in the blood is related to the incidence and development of muscle-related disorders and can serve as a stable molecular marker for the diagnosis of muscle-related diseases.

\section{Perspectives and conclusions}

Regulation of skeletal muscle development by miRNA is becoming the focus of developmental biology studies. To date, miR-206 is the only miRNA discovered that is expressed specifically in vertebrate skeletal muscles. Numerous cell-based studies, animal experiments and human research have demonstrated that miR-206 is involved in skeletal muscle development, growth/adaptation, regeneration and muscle-related diseases. Therefore, miR-206 has become a biomarker and potential therapeutic target for skeletal muscle diseases. In-depth study of the functions of miR-206 and underlying molecular mechanisms will not only provide a novel theoretical basis for revealing the mechanisms of muscle differentiation and regeneration and muscle-related diseases but also offer new enlightenment on the application of miR-206 in the treatment of muscle-related diseases and in the improvement and breeding of livestock and poultry.

\section{Acknowledgments}

Support for this work includes funding from the National Nature Science Foundation of China (Grant No. 31171219, 81271213, 81471294, 81400023 and $81271214)$, and the Science and Technology Innovation Fund of Guangdong Medical College (Grant No. STIF 201101), and Medical Scientific Research Foundation of Guangdong Province (Grant No. A2014483) and Doctor Startup Fund of Guangdong Medical College (Grant No. XB1326 and XB1329).

\section{Competing Interests}

The authors have declared that no competing interest exists.

\section{References}

1. Buckingham M. Skeletal muscle formation in vertebrates. Curr Opin Genet Dev. 2001; 11: 440-8.

2. Perry RL, Rudnick MA. Molecular mechanisms regulating myogenic determination and differentiation. Front Biosci. 2000; 5: D750-67.

3. Berkes CA, Tapscott SJ. MyoD and the transcriptional control of myogenesis. Elsevier. 2005: 585-95.

4. Potthoff MJ, Olson EN. MEF2: a central regulator of diverse developmental programs. Development. 2007; 134: 4131-40.

5. Buckingham M, Rigby PW. Gene regulatory networks and transcriptional mechanisms that control myogenesis. Dev Cell. 2014; 28: 225-38.

6. Lee CT, Risom T, Strauss WM. Evolutionary conservation of microRNA regulatory circuits: an examination of microRNA gene complexity and conserved microRNA-target interactions through metazoan phylogeny. DNA Cell Biol. 2007; 26: 209-18.

7. Stark A, Brennecke J, Bushati N, Russell RB, Cohen SM. Animal MicroRNAs confer robustness to gene expression and have a significant impact on 3'UTR evolution. Cell. 2005; 123: 1133-46.

8. Callis TE, Deng Z, Chen JF, Wang DZ. Muscling through the microRNA world. Exp Biol Med (Maywood). 2008; 233: 131-8.
9. Chen JF, Callis TE, Wang DZ. microRNAs and muscle disorders. J Cell Sci. 2009; 122: 13-20.

10. Sempere LF, Freemantle S, Pitha-Rowe I, Moss E, Dmitrovsky E, Ambros V. Expression profiling of mammalian microRNAs uncovers a subset of brain-expressed microRNAs with possible roles in murine and human neuronal differentiation. Genome Biol. 2004; 5: R13.

11. van Rooij E, Quiat D, Johnson BA, Sutherland LB, Qi X, Richardson JA, et al. A family of microRNAs encoded by myosin genes governs myosin expression and muscle performance. Dev Cell. 2009; 17: 662-73.

12. Small EM, O'Rourke JR, Moresi V, Sutherland LB, McAnally J, Gerard RD, et al. Regulation of PI3-kinase/Akt signaling by muscle-enriched microRNA-486. Proc Natl Acad Sci U S A. 2010; 107: 4218-23.

13. van Rooij E, Sutherland LB, Qi X, Richardson JA, Hill J, Olson EN. Control of stress-dependent cardiac growth and gene expression by a microRNA. Science. 2007; 316: 575-9.

14. McCarthy JJ. MicroRNA-206: the skeletal muscle-specific myomiR. Biochim Biophys Acta. 2008; 1779: 682-91.

15. Amirouche A, Tadesse H, Miura P, Belanger G, Lunde JA, Cote J, et al. Converging pathways involving microRNA-206 and the RNA-binding protein KSRP control post-transcriptionally utrophin A expression in skeletal muscle. Nucleic Acids Res. 2014; 42: 3982-97.

16. Anderson C, Catoe H, Werner R. MIR-206 regulates connexin43 expression during skeletal muscle development. Nucleic Acids Res. 2006; 34: 5863-71.

17. McCarthy JJ, Esser KA. MicroRNA-1 and microRNA-133a expression are decreased during skeletal muscle hypertrophy. J Appl Physiol (1985). 2007; 102: 306-13.

18. Takada S, Berezikov E, Yamashita Y, Lagos-Quintana M, Kloosterman WP, Enomoto $\mathrm{M}$, et al. Mouse microRNA profiles determined with a new and sensitive cloning method. Nucleic Acids Res. 2006; 34: e115.

19. Sweetman D, Goljanek K, Rathjen T, Oustanina S, Braun T, Dalmay T, et al. Specific requirements of MRFs for the expression of muscle specific microRNAs, miR-1, miR-206 and miR-133. Dev Biol. 2008; 321: 491-9.

20. Winbanks CE, Wang B, Beyer C, Koh P, White L, Kantharidis P, et al. TGF-beta regulates miR-206 and miR-29 to control myogenic differentiation through regulation of HDAC4. J Biol Chem. 2011; 286: 13805-14

21. Rachagani S, Cheng Y, Reecy JM. Myostatin genotype regulates muscle-specific miRNA expression in mouse pectoralis muscle. BMC Res Notes. 2010; 3: 297.

22. Cesana M, Cacchiarelli D, Legnini I, Santini T, Sthandier O, Chinappi M, et al. A long noncoding RNA controls muscle differentiation by functioning as a competing endogenous RNA. Cell. 2011; 147: 358-69.

23. Rao PK, Kumar RM, Farkhondeh M, Baskerville S, Lodish HF. Myogenic factors that regulate expression of muscle-specific microRNAs. Proc Natl Acad Sci U S A. 2006; 103: 8721-6.

24. Gagan J, Dey BK, Layer R, Yan Z, Dutta A. Notch3 and Mef2c proteins are mutually antagonistic via Mkp1 protein and miR-1/206 microRNAs in differentiating myoblasts. J Biol Chem. 2012; 287: 40360-70.

25. Kim HK, Lee YS, Sivaprasad U, Malhotra A, Dutta A. Muscle-specific microRNA miR-206 promotes muscle differentiation. J Cell Biol. 2006; 174: 677-87.

26. Kassar-Duchossoy L, Giacone E, Gayraud-Morel B, Jory A, Gomes D, Tajbakhsh S. Pax3/Pax7 mark a novel population of primitive myogenic cells during development. Genes Dev. 2005; 19: 1426-31.

27. Zammit PS, Relaix F, Nagata Y, Ruiz AP, Collins CA, Partridge TA, et al. Pax7 and myogenic progression in skeletal muscle satellite cells. J Cell Sci. 2006; 119: 1824-32.

28. Buckingham M, Bajard L, Daubas P, Esner M, Lagha M, Relaix F, et al. Myogenic progenitor cells in the mouse embryo are marked by the expression of Pax3/7 genes that regulate their survival and myogenic potential. Anat Embryol (Berl). 2006; 211 Suppl 1: 51-6.

29. Chen JF, Tao Y, Li J, Deng Z, Yan Z, Xiao X, et al. microRNA-1 and microRNA-206 regulate skeletal muscle satellite cell proliferation and differentiation by repressing Pax7. J Cell Biol. 2010; 190: 867-79.

30. Hirai H, Verma M, Watanabe S, Tastad C, Asakura Y, Asakura A. MyoD regulates apoptosis of myoblasts through microRNA-mediated down-regulation of Pax3. J Cell Biol. 2010; 191: 347-65.

31. Araya R, Eckardt D, Maxeiner S, Kruger O, Theis M, Willecke K, et al. Expression of connexins during differentiation and regeneration of skeletal muscle: functional relevance of connexin43. J Cell Sci. 2005; 118: 27-37.

32. Rosenberg MI, Georges SA, Asawachaicharn A, Analau E, Tapscott SJ. MyoD inhibits Fstl1 and Utrn expression by inducing transcription of miR-206. J Cell Biol. 2006; 175: 77-85.

33. Boutz PL, Chawla G, Stoilov P, Black DL. MicroRNAs regulate the expression of the alternative splicing factor $\mathrm{nPTB}$ during muscle development. Genes Dev. 2007; 21: 71-84.

34. Liu H, Chen SE, Jin B, Carson JA, Niu A, Durham W, et al. TIMP3: a physiological regulator of adult myogenesis. J Cell Sci. 2010; 123: 2914-21.

35. Chang NC, Rudnicki MA. Satellite cells: the architects of skeletal muscle. Curr Top Dev Biol. 2014; 107: 161-81.

36. Kang JS, Krauss RS. Muscle stem cells in developmental and regenerative myogenesis. Curr Opin Clin Nutr Metab Care. 2010; 13: 243-8.

37. Dey BK, Gagan J, Dutta A. miR-206 and -486 induce myoblast differentiation by downregulating Pax7. Mol Cell Biol. 2011; 31: 203-14. 
38. Nakasa $T$, Ishikawa $M$, Shi M, Shibuya $H$, Adachi N, Ochi M. Acceleration of muscle regeneration by local injection of muscle-specific microRNAs in rat skeletal muscle injury model. J Cell Mol Med. 2010; 14: 2495-505.

39. Sandri M. Signaling in muscle atrophy and hypertrophy. Physiology (Bethesda). 2008; 23: 160-70.

40. Yan B, Zhu CD, Guo JT, Zhao LH, Zhao JL. miR-206 regulates the growth of the teleost tilapia (Oreochromis niloticus) through the modulation of IGF-1 gene expression. J Exp Biol. 2013; 216: 1265-9.

41. Winbanks CE, Beyer C, Hagg A, Qian H, Sepulveda PV, Gregorevic P. miR-206 represses hypertrophy of myogenic cells but not muscle fibers via inhibition of HDAC4. PLoS One. 2013; 8: e73589.

42. Yuasa K, Hagiwara Y, Ando M, Nakamura A, Takeda S, Hijikata T. MicroRNA-206 is highly expressed in newly formed muscle fibers: implications regarding potential for muscle regeneration and maturation in muscular dystrophy. Cell Struct Funct. 2008; 33: 163-9.

43. Schuelke M, Wagner KR, Stolz LE, Hubner C, Riebel T, Komen W, et al. Myostatin mutation associated with gross muscle hypertrophy in a child. $\mathrm{N}$ Engl J Med. 2004; 350: 2682-8.

44. Kambadur R, Sharma M, Smith TP, Bass JJ. Mutations in myostatin (GDF8) in double-muscled Belgian Blue and Piedmontese cattle. Genome Res. 1997; 7: 910-6.

45. McPherron AC, Lawler AM, Lee SJ. Regulation of skeletal muscle mass in mice by a new TGF-beta superfamily member. Nature. 1997; 387: 83-90.

46. Clop A, Marcq F, Takeda H, Pirottin D, Tordoir X, Bibe B, et al. A mutation creating a potential illegitimate microRNA target site in the myostatin gene affects muscularity in sheep. Nat Genet. 2006; 38: 813-8.

47. Nallamilli BR, Ankala A, Hegde M. Molecular diagnosis of duchenne muscular dystrophy. Curr Protoc Hum Genet. 2014; 83: 9251-99.

48. Greco S, De Simone M, Colussi C, Zaccagnini G, Fasanaro P, Pescatori M, et al. Common micro-RNA signature in skeletal muscle damage and regeneration induced by Duchenne muscular dystrophy and acute ischemia. FASEB J. 2009; 23: $3335-46$

49. Liu N, Williams AH, Maxeiner JM, Bezprozvannaya S, Shelton JM, Richardson JA, et al. microRNA-206 promotes skeletal muscle regeneration and delays progression of Duchenne muscular dystrophy in mice. J Clin Invest. 2012; 122: 2054-65.

50. Basu U, Lozynska O, Moorwood C, Patel G, Wilton SD, Khurana TS. Translational regulation of utrophin by miRNAs. PLoS One. 2011; 6: e29376.
51. Moorwood C, Soni N, Patel G, Wilton SD, Khurana TS, A cell-based high-throughput screening assay for posttranscriptional utrophin upregulation. J Biomol Screen. 2013; 18: 400-6.

52. McCarthy JJ, Esser KA, Andrade FH. MicroRNA-206 is overexpressed in the diaphragm but not the hindlimb muscle of mdx mouse. Am J Physiol Cell Physiol. 2007; 293: C451-7.

53. Taulli R, Bersani F, Foglizzo V, Linari A, Vigna E, Ladanyi M, et al. The muscle-specific microRNA miR-206 blocks human rhabdomyosarcoma growth in xenotransplanted mice by promoting myogenic differentiation. J Clin Invest. 2009; 119: 2366-78.

54. Macquarrie KL, Yao Z, Young JM, Cao Y, Tapscott SJ. miR-206 integrates multiple components of differentiation pathways to control the transition from growth to differentiation in rhabdomyosarcoma cells. Skelet Muscle. 2012; 2: 7.

55. Brown RH. Medicine. A reinnervating microRNA. Science. 2009; 326: 1494-5.

56. Jeng SF, Rau CS, Liliang PC, Wu CJ, Lu TH, Chen YC, et al. Profiling muscle-specific microRNA expression after peripheral denervation and reinnervation in a rat model. J Neurotrauma. 2009; 26: 2345-53.

57. Miura P, Amirouche A, Clow C, Belanger G, Jasmin BJ. Brain-derived neurotrophic factor expression is repressed during myogenic differentiation by miR-206. J Neurochem. 2012; 120: 230-8.

58. Toivonen JM, Manzano R, Olivan S, Zaragoza P, Garcia-Redondo A, Osta R. MicroRNA-206: a potential circulating biomarker candidate for amyotrophic lateral sclerosis. PLoS One. 2014; 9: e89065.

59. Hu J, Kong M, Ye Y, Hong S, Cheng L, Jiang L. Serum miR-206 and other muscle-specific microRNAs as non-invasive biomarkers for Duchenne muscular dystrophy. J Neurochem. 2014; 129: 877-83.

60. Zaharieva IT, Calissano M, Scoto M, Preston M, Cirak S, Feng L, et al. Dystromirs as serum biomarkers for monitoring the disease severity in Duchenne muscular Dystrophy. PLoS One. 2013; 8: e80263.

61. Li X, Li Y, Zhao L, Zhang D, Yao X, Zhang H, et al. Circulating Muscle-specific miRNAs in Duchenne Muscular Dystrophy Patients. Mol Ther Nucleic Acids. 2014; 3: e177.

62. Mizuno H, Nakamura A, Aoki Y, Ito N, Kishi S, Yamamoto K, et al. Identification of muscle-specific microRNAs in serum of muscular dystrophy animal models: promising novel blood-based markers for muscular dystrophy. PLoS One. 2011; 6: e18388. 